\title{
Role of laparoscopy in the treatment of patients with endometrial cancer
}

\author{
Roberto Tozzi • Riccardo Garruto Campanile
}

Received: 7 May 2007 / Accepted: 14 June 2007 / Published online: 28 July 2007

(C) Springer-Verlag 2007

\begin{abstract}
Patients with endometrial cancer (EC) are conventionally staged by surgery. Depending on the tumor risk factors and the surgical findings, adjuvant treatment is indicated in the form of radiotherapy or chemotherapy. The FIGO guidelines on surgical staging are clear on the importance of assessing the presence of extra-uterine spread of disease, but are elusive on how extensive the surgical staging should be. Also, the role of lymphadenectomy and adjuvant radiotherapy in these patients is the object of controversy, as confirmed by recent results of clinical trials. With surgery remaining the cornerstone of treatment, the surgical approach associated with the least complications should be pursued, particularly since the association of surgery and radiotherapy can escalate the overall treatment morbidity. Therefore, in the last 15 years, laparoscopy has slowly been replacing the traditional midline laparotomy. The results of the few clinical trials and several retrospective studies are unanimous. The laparoscopic approach is feasible, safe and effective and has a lower complication rate as compared to laparotomy in all patients. The number of patients with endometrial cancer included in published studies so far is too low to achieve statistical significance with respect to survival outcomes. However, for some groups of patients, there are results from clinical trials showing that laparoscopy is the method of choice in view of the outstanding reduced surgical morbidity.
\end{abstract}

Keywords Endometrial · Cancer · Surgey · Laparoscopy

\footnotetext{
R. Tozzi $(\bowtie) \cdot R$. Garruto Campanile

Department of Gynaecologic Oncology and Mini-invasive

Surgery, IRCCS Humanitas Clinical Institute,

Via Manzoni 56,

20089 Rozzano, Milan, Italy

e-mail: roberto.tozzi@humanitas.it
}

\section{Introduction, materials and methods}

Endometrial cancer (EC) is still the fourth most common gynecologic malignancy, affecting mostly postmenopausal women of the western countries. Although two different patterns of development have been distinguished, the most common and best characterised type of EC arises in women with unopposed exposition to estrogens. Such a situation stimulates the endometrium, which passes through a phase of hyperplasia and ends with transformation into invasive cancer. Patients with EC are often obese, diabetic and suffer from hypertension [1]. Obesity is, however, the only factor independently increasing the risk of developing EC [2-4], probably because the estrogen production that takes place in the abundant adipose tissue of obese women is not balanced by a correspondent progesterone production.

Since 1988, FIGO has established a classification system based on the surgical findings that mandates an accurate exploration of the abdomen, peritoneal washing, total hysterectomy, bilateral salpingo-ophorectomy and evaluation of the lymph nodes [5].

Precise guidelines on the extension of the lymphadenectomy are lacking, particularly on which patients should undergo this procedure, whether it should be a simple sampling or a systemic lymphadenectomy and finally if it should be limited to the pelvis or should involve the paraaortic lymph nodes. The decision is left "incumbing on the clinician...to define those cases that can be managed readily and those cases that will require greater surgical expertise and training" [5]. The other area of controversy is the indication for adjuvant radiotherapy, especially after the results of two clinical trials have recently been published [6, 7]. Unfortunately, no clinical trial has so far showed an improved overall survival regardless of using lymphadenectomy or radiotherapy. The practice of lymphadenectomy 
provides relevant information on the lymph-nodal status, limiting the radiotherapy to patients with positive lymph nodes. On the other hand, elective adjuvant external beam radiotherapy (EBR) would avoid the need for pelvic lymphadenectomy. Although no survival benefit has been proved so far by combining treatment modalities, a striking increase in the morbidity has been shown in patients undergoing a formal pelvic and/or para-aortic lymphadenectomy followed by EBR. As a result of these unresolved controversies, the clinical practice is variable, and the literature reports on patients treated with both lymphadenectomy and radiotherapy, even if no benefit has been shown. The only effective attempt to reduce the morbidity of a treatment of patients with EC that has otherwise been unchanged for 20 years is the replacement of the traditional midline laparotomy (LPT) with a laparoscopic-vaginal (LPS) approach. This was started about 15 years ago, but has only slowly and recently achieved consensus. Current data, mainly produced by retrospective reports and by few clinical trials, are providing evidence for the feasibility, safety and efficacy of LPS and also for the lower rate of complications as compared to LPT [8-23]. This review will analyse the data published so far in the literature, determining variables shared in the different studies and comparing these with control groups. The aim is to define the evidence for the use of LPS in patients with EC.

\section{Results}

Since the very first paper on re-staging of patients with EC [8] in 1994, the laparoscopic treatment of patients with EC has been described in several studies, including a prospective randomised trial comparing LPS to LPT [19]. As this last study was conducted in a single institution, the number of patients recruited so far is insufficient for statistical significance with respect to survival outcomes. On the contrary, some significant evidence has been provided on morbidity outcomes, especially for a subgroup of patients. A large multicentric trial was launched some years ago, called GOG LAP 2, aiming to recruit over 2,500 patients with EC to be treated by LPS or LPT. This study clearly has the power to provide valid data on survival outcomes, the main endpoint in oncologic studies. So far, 14 retrospective studies and 1 clinical trial have reported on overall 886 patients with EC treated by LPS [9-23]. Ten out of 14 reports used a case-control model, with a control group of overall 912 patients undergoing laparotomy. However, two studies alone reported on more than half of the overall patients undergoing laparotomy. They were using historical series of patients who had undergone laparotomy as the control group, in one study [21] largely exceeding the group of patients undergoing laparoscopy (251 vs. 69).
The other 4 studies of the 14 describe their experience in treating patients with EC by laparoscopy with no control group. We have selected studies including more than 50 patients per group, or at least 100 patients in studies with a single group, which leaves 9 studies out of 15 [14-23].

The surgical techniques were very similar, with only minor differences related to a total laparoscopic or laparoscopic-assisted vaginal approach. However, the extension of the surgery was different, with only few studies defining guidelines on the use of pelvic and para-aortic lymphadenectomy. In four out of nine studies the patients routinely underwent para-aortic lymphadenectomy, and the number of lymph nodes was clearly reported. In the other five studies the para-aortic lymph nodes were not sampled or no number of lymph nodes was reported. The number of pelvic lymph nodes is always reported: the yield was a mean of 15.1 lymph nodes (range 7-27) in the LPS group and 14 lymph nodes (range 5-23) in the LPT group. The mean number of para-aortic lymph nodes yielded was 6.5 (range 2.5-12) in the LPS group and 6 (range 3.5-10) in the LPT groups. The conversion rate from laparoscopy to laparotomy ranges between $1.5 \%$ and $36.5 \%$, the latter probably affected by the number of obese patients recruited in the study [16]. The operative time is broadly variable, because of reports issued in different phases of the learning curve, because of the extension of the surgical staging and finally because of the number of obese patients. Therefore, the range is between 146.6 and $265 \mathrm{~min}$. The recorded complications are diverse, but all the studies correctly provide details. The accidental lesion of small or large bowel was reported in five out of nine studies, equally distributed in patients undergoing LPS and LPT, with a rate of $0.39 \%$ to $2.2 \%$. The occurrence of a bladder lesion was registered in only three studies with a rate of $1.1 \%$ to $1.7 \%$ with no difference in the treatment modality. The same as for ureter injuries, three studies reported two events in the LPS group and one in the LPT groups, with a rate of $1.7 \%$ and $1.6 \%$, respectively. Intra-operative complications involving major vessels were mainly recorded in patients undergoing laparoscopy. Only one study reported the occurrence of such a complication in $0.8 \%$ of the patients undergoing LPT, whilst five studies recorded a rate ranging between $1.3 \%$ and $14.4 \%$ in the laparoscopy group. The other four studies reported no complications interesting the major vessels at all. The intra-operative blood loss was reported in seven out of nine studies, with a mean of $280 \mathrm{cc}$ (range of mean 200-361) in the LPS group patients. This range compares favourably with the control group of patients undergoing LPT, with an estimated mean blood loss of $405 \mathrm{cc}$ (range of mean 216-594), with all the studies reporting reduced blood loss in the LPS group. Also the blood transfusion rate was slightly lower in the patients of the LPS groups as reported in all but one study, with a rate 
ranging between $1.5 \%$ and $19.2 \%$ and a mean rate of $6 \%$ in the LPS group and a rate between $2.2 \%$ and $18.6 \%$ with a mean rate of $7.5 \%$ in the LPT group. In all the studies the length of hospitalisation was shorter for the LPS group, with a mean of 3.5 days (range $2.5-10.2$ ) vs. a mean of 7.2 days (range 4.5-15.5) in the LPT group. The occurrence of ileus was also clearly lower in the LPS group. Only one study reported a $1 \%$ rate of ileus in the LPS group patients as compared to all the studies recording the occurrence of ileus in patients undergoing LPT, ranging between $1.6 \%$ and $21 \%$, with a mean of $9.6 \%$. Quite remarkable is the difference in wound infections and dehiscence quoted in six out of nine studies: the LPS group patients experienced wound complications in $3 \%$ to $9 \%$ of the cases concentrated on, however, in three out of six studies. All six studies reported on wound complications for the patients undergoing LPT, with a range between $9 \%$ and $22 \%$. Finally, the rate of thrombosis or embolism was reported in six studies, with five out of six only reporting on events occurring to patients in the LPT group, with a range between $2.2 \%$ and $6.7 \%$. The rate of patients requiring re-operation to manage complications was reported in four out of nine studies. The rate was higher in the LPT group, with a range of $4.4 \%$ to $7.5 \%$, while only two reports observed a $1.4 \%$ rate of re-operation in the LPS group. Lymph edema or lymph cyst were more commonly occurring in the LPT group, with a range of 2.2 to $5.4 \%$ vs. 1.8 to $2.7 \%$. The costs of the two techniques were compared in two of the reviewed studies. Despite higher operative costs, the overall costs were lower in the LPS group, in view of the substantial reduction of the hospitalisation length. In the studies with a control group it appears that an overall benefit for the LPS group is present, but is not consistent between the studies with respect to single variables. The benefit reaches statistical significance only for intra-operative blood loss, length of hospitalisation and the occurrence of postoperative ileus, wound complications and thrombo-embolic events. Recently a clinical trial has reported on the morbidity outcomes of the surgical treatment for patients with EC comparing LPS to LPT. Within the recruited patients, a subgroup of women with comorbidity was identified. These patients were considered to be at high surgical risk. Comparing these sub-groups of patients for their overall risk of intra-operative, early and late post-operative complications, a multivariate analysis could identify only a the surgical intention to treat, i.e., LPS vs. LPT, as a statistically significant risk factor. Patients undergoing a laparoscopic-vaginal approach were found to have a $44 \%$ relative risk of overall complications compared to patients undergoing laparotomy.

Five studies provide data on survival outcomes. Three of the five provide median follow-up for the overall population, while the other two have a clearly shorter follow-up period for the LPS group. The survival figures are very similar among the groups, with overall survival ranging between $75 \%$ and $84 \%$ and the recurrence rate between 4 and $14 \%$. Two studies presented cause-specific survival for patients with stage I disease, ranging between $93.6 \%$ and $96 \%$. In none of the studies was a statistically significant difference found between the two groups. Also, none of the studies provides 5-year follow-up data, with the longest median follow-up being 44 months.

\section{Discussion}

The management of patients with endometrial cancer is still much debated. The role of lymphadenectomy and adjuvant radiotherapy in patients with stage I has not yet been defined. Large clinical trials have been conducted and published, leaving, however, still unanswered questions. As clear guidelines are lacking, the clinical practice offers a broad scenario of treatment modalities, including the combination of adjuvant radiotherapy following lymphadenectomy. Although no survival benefit has been proved so far, the combination of treatment modalities significantly increases the morbidity. In the contest of a pretty much unchanged therapy over the last 20 years, the only real new approach is laparoscopy, either combined with the vaginal surgery or as a total laparoscopic approach.

So far, with the exception of one clinical trial, only retrospective studies have been published. The results of the studies unanimously lead to the conclusion that the laparoscopic approach is a valid alternative to the standard of care, which for many years has been a laparotomy. In addition, the morbidity of patients undergoing the laparoscopic approach is reduced and the recovery time shortened. Looking at all these studies together, the variables that are significantly improved are the intra-operative blood loss, the length of hospitalisation and the occurrence of postoperative ileus, wound complications and thromboembolic events. The only clinical trial currently produced has provided definite data only with respect to morbidity outcomes. These latter data based on an adequate sample size have shown significant reduction in the morbidity outcomes for all the patients undergoing LPS. Additionally, a subgroup of patients with co-morbid factors has been identified that significantly profits from the LPS. For this subgroup of patients, LPS should be considered the standard of care.

However, the main outcome in oncology is survival. All the studies presented so far have provided encouraging results on this matter. None of them has shown significant difference in survival between patients undergoing LPS vs. LPT. Although this body of retrospective data would suggest that the results are similar, the conclusion that laparoscopic treatment of patients with endometrial cancer 
is equivalent to the laparotomic approach needs to be based on further results. These would be probably provided by the GOG LAP 2 trial, which has a large sample size. If this or other clinical trials confirm the good results already published, the use of laparoscopy should be adopted in the standard management of patients with endometrial cancer.

\section{References}

1. Smith RA, von Eschenbach AC, Wender et al. (2001) American Cancer Society guidelines for the early detection of cancer: update of early detection guidelines for prostate, colorectal, and endometrial cancers. CA Cancer J Clin 51:38-75

2. Furger AS, Thune I (2003) Metabolic abnormalities (hypertension, hyperglycemia and overweight), lifestyle (high energy intake and physical inactivity) and endometrial cancer risk in a Norwegian cohort. Int J Cancer 104:6, 669-676

3. Kaaks R, Lukanova AK, Kurzer M (2002) Obesity, endogenous hormones and endometrial cancer risk: a synthetic review. Cancer Epidemiol Biomark Prev 11:1531-1543

4. Roos LL, Sharp SM, Cohen MM, Wajida A (1989) Risk adjustment in claims-based research: the search for efficient approaches. J Clin Epidemiol 42:1193-1206

5. Announcements: FIGO stages-1988 Revision. Gynecol Oncol 35:125-127

6. Creutzberg CL, van Putten WLJ, Koper PCM, Lybeert MLM, Jobsen JJ, Warlam-Rodenhuis CC, Winter KAJ, Lutgens LCHW, van den Bergh ACM, van de Steen-Banasik E, Berman H, van Lent M (2000) Surgery and postoperative radiotherapy versus surgery alone for patients with stage-1 endometrial carcinoma: multicentre randomized trial. Lancet 355:1404-1411

7. Keys HM, Roberts JA, Brunetto VL, Zaino R, Spirtos NM, Bloss JD, Perlman A, Maiman MA, Bell JG (2004) A phase III trial of surgery with or without adjunctive external pelvic radiation therapy in intermediate-risk endometrial adenocarcinoma: a Gynecologic Oncology Group study. Gynecol Oncol 92(3):744751, Mar

8. Childers JM, Spirtos NM, Brainard P, Surwit EA (1994) Laparoscopic staging of the patient with incompletely staged early adenocarcinoma of the endometrium. Obstet Gynecol 83:597-600

9. Eltabbakh GH, Shamonki MI, Moody JM, Garafano LL (2002) Analysis of survival after laparoscopy in women with endometrial carcinoma. Cancer 95:1894-1901

10. Zapico A, Fuentes P, Grassa A, Arnanz F, Otazua J, Cortes-Prieto J (2005) Laparoscopic-assisted vaginal hysterectomy versus abdom- inal hysterectomy in stages I and II endometrial cancer. Operating data, follow up and survival. Gynecol Oncol 98:222-227

11. Spirtos NM, Schlaerth JB, Gross GM, Spirtos TW, Schlaerth AC, Ballon SC (1996) Cost and quality of life analyses of surgery for early endometrial cancer: laparotomy vs laparoscopy. Am J Obstet Gynecol 174:1795-1800

12. Langebrekke A, Istre O, Hallqvist AC, Hartgill TW, Onsrud M (2002) Comparison of laparoscopy and laparotomy in patients with endometrial cancer. J Am Assoc Gynecol Laparosc 9(2):152157, May

13. Kuoppala T, Tomas E, Heinonen PK (2004) Clinical outcome and complications of laparoscopic surgery compared with traditional surgery in women with endometrial cancer. Arch Gynecol Obstet 270(1):25-30, Jul

14. Magrina JF, Mutone NF, Weaver AL, Magtibay PM, Fowler RS, Cornella JL (1999) Laparoscopic lymphadenectomy and vaginal or laparoscopic hysterectomy with bilateral salpingo-oophorectomy for endometrial cancer: morbidity and survival. Am J Obstet Gynecol 181:376-381

15. Eltabbakh GH, Shamonki MI, Moody JM, Garafano LL (2000) Hysterectomy for obese women with endometrial cancer: laparoscopy or laparotomy? Gynecol Oncol 78:329-335

16. Scribner DR, Walker JL, Johnson GA, McMeekin DS, Gold MA, Mannel RS (2002) Laparoscopic pelvic and paraaortic lymphnode dissection in the obese. Gynecol Oncol 84:426-430

17. Eltabbakh GH, Shamonki MI, Moody JM, Garafano LL (2001) Laparoscopy as the primary modality for the treatment of women with endometrial carcinoma. Cancer 91:378-387

18. Tozzi R, Malur S, Köhler C, Schneider A (2005) Laparoscopy vs. laparotomy in endometrial cancer: first analysis of survival of a randomized prospective study. J Minim Invasive Gynecol 12 (2):130-136, Mar-Apr

19. Tozzi R, Malur S, Köhler C, Schneider A (2005) Analysis of morbidity in patients with endometrial cancer: is there a commitment to offer laparoscopy? Gynecol Oncol 97(1):4-9, Apr

20. Scribner DR Jr, Walker JL, Johnson GA, McMeekin SD, Gold MA, Mannel RS (2001) Surgical management of early-stage endometrial cancer in the elderly: is laparoscopy feasible? Gynecol Oncol 83:563-568, Dec

21. Gemignani ML, Curtin JP, Zelmanovich J, Patel DA, Venkatraman E, Barakat RR (1999) Laparoscopic-assisted vaginal hysterectomy for endometrial cancer: clinical outcomes and hospital charges. Gynecol Oncol 73:5-11

22. Obermair A, Manolitsas TP, Lung Y, Hammond IG, Mc Cartney AJ (2004) Total laparoscopic hysterectomy for endometrial cancer: patterns of recurrence and survival. Gynecol Oncol 92:789-793

23. Kim DY, Kim MK, Kim JH, Suh DS, Kim YM, Kim YT, Mok JE, Nam JH. Laparoscopic-assisted vaginal hysterectomy versus abdominal hysterectomy in patients with stage I and II endometrial cancer 